\title{
Local and systemic reactogenicity of COVID-19 vaccine BNT162b2 in patients with systemic lupus erythematosus and rheumatoid arthritis
}

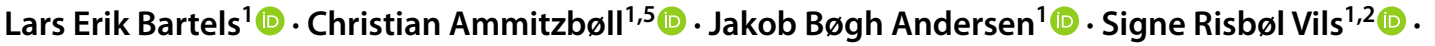 \\ Clara Elbæk Mistegaard ${ }^{1,2,5}$ - Anders Dahl Johannsen ${ }^{1}()_{0} \cdot$ Marie-Louise From Hermansen $^{1}(1)$.

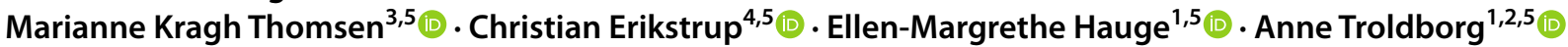

Received: 14 July 2021 / Accepted: 10 August 2021 / Published online: 2 September 2021

(c) The Author(s), under exclusive licence to Springer-Verlag GmbH Germany, part of Springer Nature 2021

\begin{abstract}
Vaccines against severe acute respiratory syndrome coronavirus 2 (SARS-CoV-2) were launched in December 2020. Vaccination of patients with rheumatic diseases is recommended, as they are considered at higher risk of severe COVID-19 than the general population. Patients with rheumatic disease have largely been excluded from vaccine phase 3 trials. This study explores the safety and reactogenicity of BNT162b2 among patients with rheumatic diseases. Patients with systemic lupus erythematosus (SLE) and rheumatoid arthritis (RA), median age 58.8 years, 285 subjects in total, were vaccinated twice with the BNT162b2 (Pfizer/BioNTech). Questionnaires on reactogenicity matching the original phase 3 study were answered seven days after completed vaccination. The majority of SLE and RA patients experienced either local (78.0\%) or systemic reactions $(80.1 \%)$. Only $1.8 \%$ experienced a grade- 4 reaction. Compared to the original study, we found more frequent fatigue [Odds ratio (OR) $2.2(1.7-2.8)$ ], headache [OR 1.7 (1.3-2.2)], muscle pain [OR 1.8 (1.4-2.3)], and joint pain [OR $2.3(1.7-3.0)]$ in patients. In contrast, the use of antipyretics was less frequent [OR $0.5(0.3-0.6)]$. Patients with SLE and RA experience reactogenicity to the Pfizer-BioNTech BNT162b2 COVID-19 vaccine. Reactogenicity was more frequent in patients, however, not more severe compared with healthy controls.
\end{abstract}

Keywords COVID-19 - Arthritis, rheumatoid $\cdot$ Lupus erythematosus, systemic $\cdot$ Patient reported $\cdot$ Outcome measures · Vaccination

Christian Ammitzbøll

chramm@rm.dk

1 Department of Rheumatology, Aarhus University Hospital, Palle Juul-Jensens Boulevard 59, Entrance E, 8200 Aarhus, Denmark

2 Department of Biomedicine, Aarhus University, Aarhus, Denmark

3 Department of Clinical Microbiology, Aarhus University Hospital, Aarhus, Denmark

4 Department of Clinical Immunology, Aarhus University Hospital, Aarhus, Denmark

5 Department of Clinical Medicine, Aarhus University, Aarhus, Denmark

\section{Introduction}

World Health Organization declared infection with severe acute respiratory syndrome coronavirus 2 (SARS-CoV-2) as a global pandemic March 2020, and since then the world has been in a battle. The weaponry has been infection control measures, advanced supportive treatment of SARS-CoV-2 patients, and latest the development of vaccines.

American College of Rheumatology (ACR) has recommended vaccination of patients with rheumatic diseases and considers this group to be at higher risk of severe COVID-19 than the general population [1,2]. A further encouragement of vaccination has been that the COVID-19 pandemic has prompted isolation in patients with rheumatic diseases [3, 4].

The BNT162b2 is an mRNA vaccine developed by PfizerBioNTech [5]. Double vaccination with 21 days apart offers 95\% protection against COVID-19 [6]. The initial phase 3 trial reported few side effects, and the mRNA vaccination 
strategy seems promising. However, treatment with immunosuppressive therapy was a key exclusion criterion in Polack et al., and of the 21,720 participants who received the vaccine, only 62 suffered from rheumatic disease, which impedes the extrapolation to clinical practice [7].

Given the limited knowledge on vaccine safety in patients with rheumatic diseases and the increased demand for such knowledge as vaccines become readily accessible, we set out to explore the reactogenicity of the BNT162b2 vaccine in patients with systemic lupus erythematosus (SLE) and rheumatoid arthritis (RA).

\section{Methods}

\section{Subjects}

Patients were recruited from the COPANARD (Corona Pandemic Autoimmune Rheumatic Disease) cohort [3]. Outpatients with SLE or RA were identified through hospital records at the Department of Rheumatology, Aarhus University Hospital, Denmark. SLE patients fulfilled the 1997 updated ACR criteria for SLE. RA patients fulfilled either the 1987 ACR or 2010 ACR/EULAR Classification Criteria and received treatment with either a biologic or small molecule disease-modifying anti-rheumatic drug (DMARD). All patients fully vaccinated on July 1st 2021 were included. Disease characteristics, treatment and Charlson Comorbidity Index were obtained from the electronic health record.

\section{Patient and public involvement}

Four patient research partners and advisers from The Danish Rheumatism Association collaborated in study planning to ensure the patient perspective and results will be communicated through member network sources.

\section{Vaccination}

Patients were, following their consent, offered participation in the study if they were to accept the SARS-CoV-2 vaccine through the national vaccination program. Patients were vaccinated twice with BNT162b2 (Pfizer/BioNTech).

\section{Questionnaires on local and systemic reactions}

Seven days after the second vaccination, the patients reported local and systemic reactogenicity through an electronic questionnaire in accordance with reactions reported in the original paper [6].

Pain at the injection site was measured according to the following scale: mild, does not interfere with activity; moderate, interferes with activity; severe, prevents daily activity; and grade 4, emergency department visit or hospitalization. Likewise, redness and swelling were measured as: mild, $2.0-5.0 \mathrm{~cm}$ in diameter; moderate, $>5.0-10.0 \mathrm{~cm}$ in diameter; severe, $>10.0 \mathrm{~cm}$ in diameter; and grade 4 , necrosis or exfoliative dermatitis (for redness) and necrosis (for swelling). Fever categories were defined as follows: no fever $<38.0 \mathrm{C} ; 38.0-38.4 ; 38.5-38.9 ; 39.0-39.9 ;+40.0$ C. Additional scales were as in the original paper: fatigue, headache, chills, new or worsened muscle pain, new or worsened joint pain (mild: does not interfere with activity; moderate: some interference with activity; or severe: prevents daily activity), vomiting (mild: 1-2 times in $24 \mathrm{~h}$; moderate: $>2$ times in $24 \mathrm{~h}$; or severe: requires intravenous hydration), and diarrhea (mild: 2-3 loose stools in $24 \mathrm{~h}$; moderate: $4-5$ loose stools in $24 \mathrm{~h}$; or severe: 6 or more loose stools in $24 \mathrm{~h}$ ). Grade 4 for all events indicated an emergency department visit or hospitalization. Medication use was defined as paracetamol or non-steroid anti-inflammatory drugs and was not graded.

\section{Statistics}

All values reported are medians with interquartile range (IQR) unless otherwise stated. The statistical significance of differences was assessed using the Mann-Whitney nonparametric test for continuous variables and Pearson's chisquare test for categorical variables. Odds ratios for local and systemic reactions were calculated using data from the phase 3 trial [6]. We assumed the same distribution of age in the main safety population $(n=37.704,42.2 \%,>55$ years $)$ as in the reactogenicity subset $(n=8,183)$ [6]. The frequency of reactions used in the calculations was based on the group of $>55$ years of age at receival of the second vaccination dose.

\section{Ethics}

The Central Denmark Region Committee on Health Research Ethics was consulted concerning the present study which was performed according to Danish legislation (Ref. nr. 1-10-72-1-21). The project was approved by The Danish Data Protection Agency (1-16-02-19-21). All participants gave written informed consent. The study was performed according to the principles of the Helsinki declaration.

\section{Results}

\section{Patient characteristics}

In this study, 128 patients with SLE and 154 with RA participated. The 282 patients were primarily female (79.1\%) with a median age of $58.8(46.5-67.5)$ years 
and Charlson comorbidity score of 3. Nearly all (93.3\%) patients received immunomodulatory medicine. Compared with SLE patients, RA patients were older (63.4 vs. 53.8, $p<0.01)$ and more frequently current or past smokers (65.1 vs. $44.1 \%, p=0.01$ ); for detailed patient characteristics, see Table 1 . Median time between vaccinations

Table 1 Patient characteristics

\begin{tabular}{|c|c|c|c|c|}
\hline & SLE & & RA & \\
\hline Patients included & 128 & $(45.4)$ & 154 & $(54.6)$ \\
\hline Female sex & 113 & $(88.3)$ & 110 & $(71.4)$ \\
\hline Age, years & 53.8 & $(41.4-62.8)$ & 63.4 & $(56.0-71.2)$ \\
\hline Body mass index, $\mathrm{kg} / \mathrm{m}^{2}$ & 24.8 & $(21.8-28.4)$ & 26.3 & $(23.0-29.4)$ \\
\hline Disease duration, years & 14.5 & $(7.0-27.0)$ & 15.5 & $(7.0-22.0)$ \\
\hline Charlson comorbidity score & 2 & $(1-3)$ & 3 & $(2-4)$ \\
\hline Active/previous/never smoker, $\%$ & $8.7 / 35.4 / 55.9$ & & $12.1 / 53.0 / 34.9$ & \\
\hline \multicolumn{5}{|l|}{ Rheumatoid arthritis (RA) } \\
\hline Anti-CCP positivity & - & - & $117 / 153$ & $(76.5)$ \\
\hline IgM-RF positivity & - & - & 107 & $(69.5)$ \\
\hline Erosive disease on X-ray & - & - & 122 & $(79.2)$ \\
\hline \multicolumn{5}{|l|}{ Systemic Lupus Erythematosus (SLE) } \\
\hline \multicolumn{5}{|l|}{ ACR classification criteria } \\
\hline Malar rash & 81 & $(63.3)$ & - & - \\
\hline Discoid rash & 9 & $(7.0)$ & - & - \\
\hline Photosensitivity & 59 & $(46.1)$ & - & \\
\hline Oral ulcers & 34 & $(26.6)$ & - & - \\
\hline Nonerosive arthritis & 111 & $(86.7)$ & - & - \\
\hline Pleuritis or pericarditis & 37 & $(28.9)$ & - & - \\
\hline Renal disorder & 37 & $(28.9)$ & - & - \\
\hline Neurologic disorder & 10 & $(7.8)$ & - & - \\
\hline Hematologic disorder & 101 & $(78.9)$ & - & - \\
\hline Immunologic disorder & 120 & $(93.8)$ & - & - \\
\hline Positive antinuclear antibody & 126 & $(98.4)$ & - & - \\
\hline SLICC score, median (IQR) & 1 & $(0-2)$ & - & - \\
\hline \multicolumn{5}{|l|}{ Treatment } \\
\hline Methotrexate & 12 & $(9.4)$ & 82 & $(53.2)$ \\
\hline Salazopyrine & - & - & 8 & $(5.2)$ \\
\hline Hydroxychloroquine & 88 & $(68.8)$ & 2 & $(1.3)$ \\
\hline Prednisone & 49 & $(38.3)$ & 10 & $(6.5)$ \\
\hline Leflunomide & - & - & 14 & $(9.1)$ \\
\hline Azathioprine & 27 & $(21.1)$ & 2 & (1.3) \\
\hline Number of biologics tried, median & - & - & 2 & $(1-3)$ \\
\hline TNF- $\alpha$-inhibitors & - & - & 79 & $(52.7)$ \\
\hline Rituximab & 5 & $(3.9)$ & 22 & $(14.7)$ \\
\hline JAK-inhibitor & - & - & 16 & $(10.7)$ \\
\hline Anti-IL-6 & - & - & 23 & $(15.3)$ \\
\hline Abatacept & - & - & 10 & $(6.7)$ \\
\hline Mycophenolatemofetil & 22 & $(17.2)$ & - & - \\
\hline Belimumab & 5 & $(3.9)$ & - & - \\
\hline Other (privigen, tacrolimus, ixekizumab) & 5 & $(3.9)$ & 2 & $(1.3)$ \\
\hline No treatment & 17 & $(13.3)$ & 2 & $(1.3)$ \\
\hline
\end{tabular}

Table 1 shows patient characteristics of the participants. Values are given as median (IQR) or $n(\%)$

$S L E$ Systemic lupus erythematosus, $R A$ Rheumatoid Arthritis, Anti-CCP Anti-cyclic citrullinated peptide, $R F$ Rheumatoid factor, ACR American College of Rheumatology, SLICC Systemic Lupus International Collaborating Clinics, TNF Tumor Necrosis Factor, JAK Janus kinase, IL Interleukin 
was 22 days (21-24). All patients included fulfilled the questionnaire.

\section{Local reactogenicity}

As shown in Fig. 1, SLE and RA patients frequently reported local reactogenicity $(78.0 \%)$ but mainly in the category 'mild'. Patients reported more redness [20.2 vs. $7.0 \%$, OR $3.4(2.2-4.7)$ ] and swelling [26.6 vs. $7.0 \%$, OR $4.8(3.5-6.5)]$ compared with healthy controls, Table 2.

\section{Systemic events}

Any kind of systemic reactogenicity was reported by $80.1 \%$ of SLE and RA patients (Fig. 1). Patients with SLE and RA reported, compared to healthy controls, fever (OR 1.7), fatigue (OR 2.2), headache (OR 1.7), chills (OR 1.4), muscle pain (OR 1.8), joint pain (OR 2.3) and vomiting (OR 2.9) to be more common following vaccination, Table 2 .

Diarrhea (7.8 vs. $8.0 \%)$ was experienced by a similar percentage of patients and healthy controls. Patients used less antipyretic medication after vaccination compared with healthy controls ( 22.3 vs. $38.0 \%$, OR 0.5 ).

In total, five patients (1.8\%) reported grade-4 events following vaccination, defined as reactogenicity leading to an emergency department visit or hospitalization. One patient experienced high fever, chills, and fatigue; a second experienced severe joint pain; another patient experienced severe joint and muscle pain; a fourth patient suffered from severe and lasting fatigue, and finally, a fourth patient had pain at the injection site. No patients were hospitalized or died.

\section{Discussion}

This study describes local and systemic reactogenicity after completion of vaccination with the BNT162b2 mRNA COVID-19 vaccine in patients with SLE and RA. Our data showed that patients frequently experienced reactions following vaccination. As in the original phase 3 study, pain at the injection site, fatigue, and headache were the most common events. However, the majority of the reactions were in the category 'mild', and of the $1.8 \%$ who experienced a grade-4 event, none were hospitalized. Thus, our study supports that BNT162b2 is safe in patients with SLE and RA.

Nevertheless, by comparing the data presented here with the original phase 3 trial, we found that patients with SLE and RA suffered more frequently from fatigue, fever headache, chills, muscle pain, and joint pain. All symptoms are common to rheumatic patients, and we speculate that patients with SLE and RA thus are more prone to experience worsening of these events following vaccination. Alternatively, deviating vaccine reaction due to the disease itself or
DMARD use may contribute to the observed difference. Our patients reported less use of antipyretic medicine following vaccination than seen in the original study. It is plausible that since many SLE and RA patients take antipyretic medicine as part of their standard medications, they would be less prone to take extra in connection with the vaccination.

Biases may make this direct comparison uncertain. Our data may not have been collected identically as we do not have precise information on data collection from the original phase 3 trial. This study included patients from a smaller geographic area with a uniform ethnicity significantly different from the multinational cohort in Polack et al. Our cohort was older than the original study cohort, possibly biasing conclusions even though the original phase 3 study found that young people were more prone to reactogenicity.

Three previous studies have presented results on mRNA COVID-19 vaccine reactogenicity in patients with chronic rheumatic disease, all supporting this conclusion. Connoly et al. observed local and systemic reactions after the first mRNA vaccine in a cohort of 325 individuals, mainly patients with inflammatory arthritis and SLE [8]. The numerical values presented were close to those presented here but only reported after the first vaccination. With mRNA vaccines, reactogenicity is more common following the second injection [6]. In a vaccine study of 26 patients with mixed inflammatory conditions, receiving mainly BNT162b2, no statistically significant differences between healthy individuals and patients with chronic inflammatory diseases were observed following the second vaccination [9]. However, the study was conducted on a very limited number of individuals, which leaves hard conclusions open to doubt. Furer et al. recently published data on a population of patients with different rheumatic diseases $(n=686)$ from [10]. They concluded that the prevalence of mild adverse events was similar in patients and controls, and that results gave a reassurance for a good safety profile of the vaccine, with most adverse events being transient and mild [10].

The strengths of this study lie within the well-characterized patient cohort with regard to classification, treatment, and comorbidity. Further, we sought to focus on reactogenic events comparable to the original study and could therefore compare directly to the original vaccine study. Finally, our findings are unlikely to be biased by a negative attitude towards vaccination, as vaccine hesitancy is generally low in Denmark, and $92.9 \%$ of the original COPANARD cohort wished to be vaccinated $[11,12]$.

Our study has limitations. The questionnaire was translated into Danish intending to mirror the questionnaire in the original study. The questionnaire was, however, not validated. Additionally, severe and rare unwanted vaccine side effects are unlikely to occur in a patient cohort of this size and with a relatively short observation period. Lastly, it would have been ideal to compare the results with real-world 

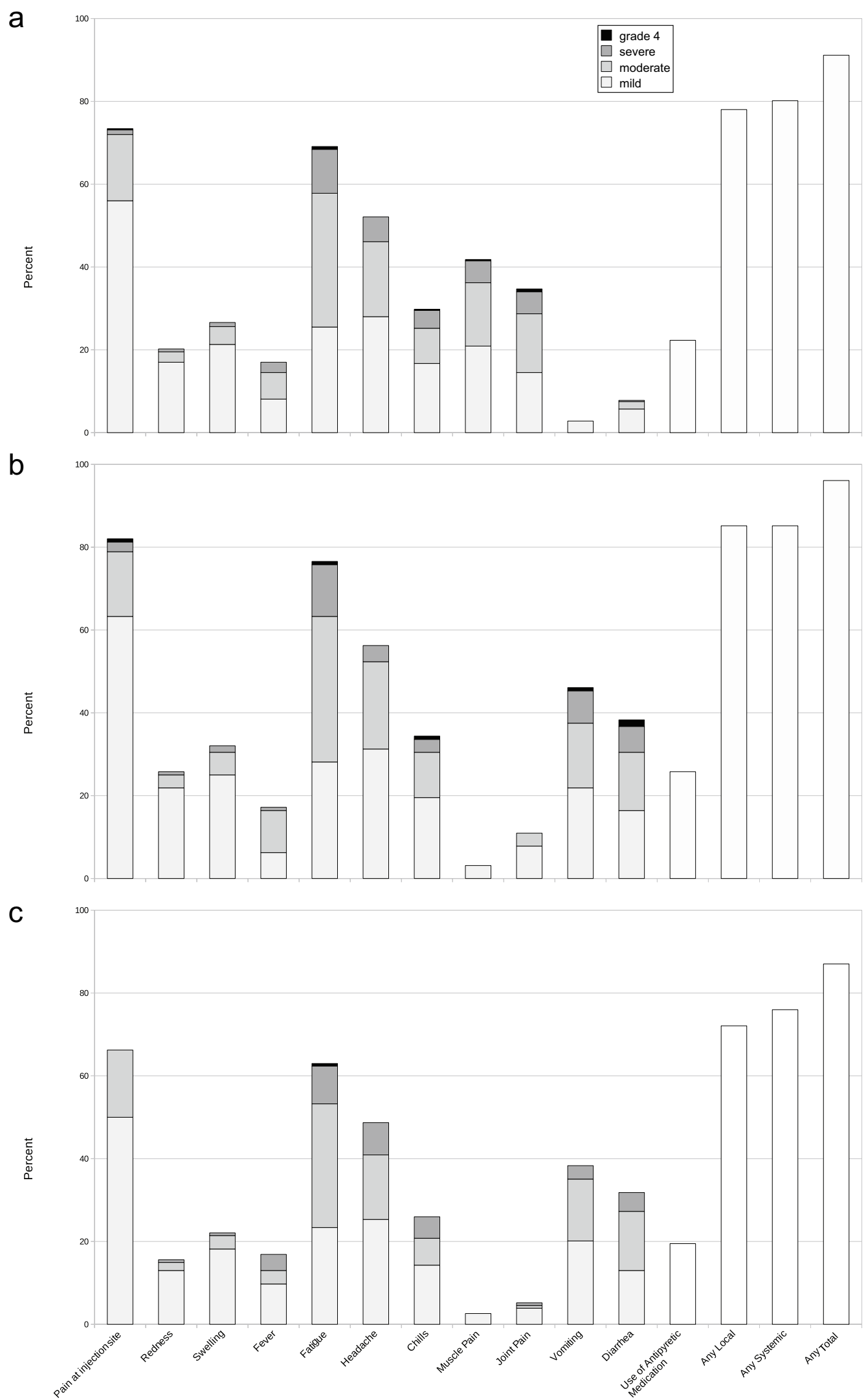

Fig. 1 Reactogenicity in percentage seven days after 2nd vaccination. A All patients, B SLE and C RA patients. Every bar represents the reported uneasiness both local and systemic. The three shades of gray represent mild, moderate, and severe reactogenicity. The black color represents grade-4 events which indicated an emergency department visit or hospitalization. The four last bars are dichotomous outcomes 
Table 2 Reactogenicity in patients compared to healthy controls

\begin{tabular}{|c|c|c|c|c|c|c|c|c|c|}
\hline & None & Mild & Moderate & Severe & Grade 4 & Total (\%) & NEJM [6] (\%) & OR & $95 \% \mathrm{CI}$ \\
\hline Pain at injection site & 26.6 & 56.0 & 16.0 & 1.1 & 0.3 & 73.4 & 66 & 1.4 & $1.1-1.9$ \\
\hline Redness & 79.8 & 17.0 & 2.5 & 0.7 & - & 20.2 & 7 & 3.4 & $2.4-4.7$ \\
\hline Swollen & 73.4 & 21.3 & 4.3 & 1.0 & - & 26.6 & 7 & 4.8 & $3.5-6.5$ \\
\hline Fever & 83.0 & 8.1 & 6.4 & 2.5 & & 17.0 & 11 & 1.7 & $1.2-2.3$ \\
\hline Fatigue & 30.9 & 25.5 & 32.3 & 10.6 & 0.7 & 69.1 & 51 & 2.2 & $1.7-2.8$ \\
\hline Headache & 47.9 & 28.0 & 18.1 & 6.0 & - & 52.1 & 39 & 1.7 & $1.3-2.2$ \\
\hline Chills & 70.2 & 16.7 & 8.5 & 4.3 & 0.3 & 29.8 & 23 & 1.4 & $1.1-1.9$ \\
\hline Muscular pain & 58.2 & 20.9 & 15.3 & 5.3 & 0.3 & 41.8 & 29 & 1.8 & $1.4-2.3$ \\
\hline Joint pain & 65.3 & 14.5 & 14.2 & 5.3 & 0.7 & 34.7 & 19 & 2.3 & $1.7-3.0$ \\
\hline Vomiting & 97.2 & 2.8 & - & - & - & 2.8 & 1 & 2.9 & $1.2-6.5$ \\
\hline Diarrhea & 92.2 & 5.7 & 1.8 & 0.3 & - & 7.8 & 8 & 1.0 & $0.6-1.5$ \\
\hline Use of antipyretic medicine & & & & & & 22.3 & 38 & 0.5 & $0.3-0.6$ \\
\hline
\end{tabular}

Table 2 shows the reactogenicity in percentage seven days after 2 nd vaccination for both SLE and RA patients $(n=285)$ and participants in the original phase 3 trial (abbreviated NEJM) [6]. The Odds Ratio (OR) is calculated between patients and participants in the original phase 3 study

data of the normal population in Denmark, however, this was not possible due to the Danish vaccine plan.

This study adds to the notion that the BNT162b2 COVID19 vaccine is safe and well tolerated in patients with SLE and RA. Prior to vaccination, clinicians can advise their patients that they should expect more frequent reactogenicity compared to healthy controls. However, they can also advise their patients that they should not fear more frequent or severe grade-4 events. Our data also suggest that patients may be reminded that anti-pyretic medicine may be useful after vaccination to restrict reactogenicity.

In conclusion, patients with SLE and RA experience reactogenicity to the Pfizer-BioNTech BNT162b2 COVID19 vaccine. Reactogenicity was more frequent in patients, however, not more severe compared with healthy controls.

Funding This study was funded by the Danish Rheumatism Association.

Data availability Does not apply to the current study.

Code availability Does not apply to the current study.

\section{Declarations}

Conflict of interest None of the authors had any conflicts of interest or competing interests.

Ethical approval The Central Denmark Region Committee on Health Research Ethics was consulted concerning the present study (Ref. nr. 1-10-72-1-21). The project was approved by The Danish Data Protection Agency (1-16-02-19-21).

Consent to participate All participants gave informed consent before participation in the present study.

\section{References}

1. Curtis JR, Johnson SR, Anthony DD et al (2021) American College of Rheumatology Guidance for COVID-19 vaccination in patients with rheumatic and musculoskeletal diseases-version 1. Arthritis Rheumatol. https://doi.org/10.1002/art.41734

2. Bower H, Frisell T, Di Giuseppe D et al (2021) Impact of the COVID-19 pandemic on morbidity and mortality in patients with inflammatory joint diseases and in the general population: a nationwide Swedish cohort study. Ann Rheum Dis. https://doi. org/10.1136/ANNRHEUMDIS-2021-219845

3. Ammitzbøll C, Andersen JB, Vils SR et al (2021) Isolation, behavioral changes and low seroprevalence of SARS-CoV-2 antibodies in patients with systemic lupus erythematosus or rheumatoid arthritis. Arthritis Care Res (Hoboken). https://doi.org/10.1002/ ACR.24716

4. van Zanten J, Fenton S, Brady S et al (2020) Mental health and psychological wellbeing in rheumatoid arthritis during COVID19-can physical activity help. Mediterr J Rheumatol. https://doi. org/10.31138/MJR.31.3.284

5. Walsh E, Frenck R, Falsey A et al (2020) Safety and immunogenicity of two RNA-based Covid-19 vaccine candidates. N Engl J Med 383:2439-2450. https://doi.org/10.1056/NEJMOA2027906

6. Polack FP, Thomas SJ, Kitchin N et al (2020) Safety and efficacy of the BNT162b2 mRNA Covid-19 vaccine. N Engl J Med 383:2603-2615. https://doi.org/10.1056/NEJMoa2034577

7. Schulze-Koops H, Specker C, Skapenko A (2021) Vaccination of patients with inflammatory rheumatic diseases against SARS-CoV-2: considerations before widespread availability of the vaccines. RMD Open. https://doi.org/10.1136/RMDOP EN-2020-001553

8. Connolly C, Ruddy J, Boyarsky B et al (2021) Safety of the first dose of mRNA SARS-CoV-2 vaccines in patients with rheumatic and musculoskeletal diseases. Ann Rheum Dis. https://doi.org/10. 1136/ANNRHEUMDIS-2021-220231

9. Geisen U, Berner D, Tran F et al (2021) Immunogenicity and safety of anti-SARS-CoV-2 mRNA vaccines in patients with chronic inflammatory conditions and immunosuppressive therapy in a monocentric cohort. Ann Rheum Dis. https://doi.org/10.1136/ ANNRHEUMDIS-2021-220272

10. Furer V, Eviatar T, Zisman D et al (2021) Immunogenicity and safety of the BNT162b2 mRNA COVID-19 vaccine in adult 
patients with autoimmune inflammatory rheumatic diseases and in the general population: a multicentre study. Ann Rheum Dis. https://doi.org/10.1136/annrheumdis-2021-220647

11. de Figueiredo A, Simas C, Karafillakis E et al (2020) Mapping global trends in vaccine confidence and investigating barriers to vaccine uptake: a large-scale retrospective temporal modelling study. Lancet 396:898-908. https://doi.org/10.1016/S01406736(20)31558-0

12. Ammitzbøll C, Thomsen MK, Erikstrup C et al (2021) National differences in vaccine hesitancy: a concern for the external validity of vaccine studies. Lancet Rheumatol. https://doi.org/10. 1016/S2665-9913(21)00083-7

Publisher's Note Springer Nature remains neutral with regard to jurisdictional claims in published maps and institutional affiliations. 